\title{
Esophageal Intraepithelial Neoplasia
}

National Cancer Institute

\section{Source}

National Cancer Institute. Esophageal Intraepithelial Neoplasia. NCI Thesaurus. Code C4839.

A lesion that is characterized by architectural and cytologic abnormalities of the esophageal epithelium, and carries a predisposition for progression to invasive carcinoma. Intraepithelial neoplasia (dysplasia) of the esophagus is graded as low or high grade. In this two-tier system, severe dysplasia and carcinoma in situ are included under the rubric of high grade intraepithelial neoplasia and may have the same clinical implications. (WHO, 2000) -- 2003 